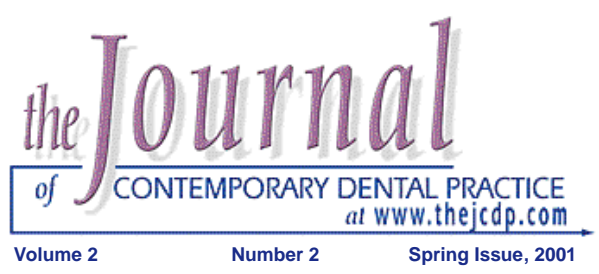

\title{
E-mail: A New Management Parameter
}

\section{James Day, DDS, MEd}

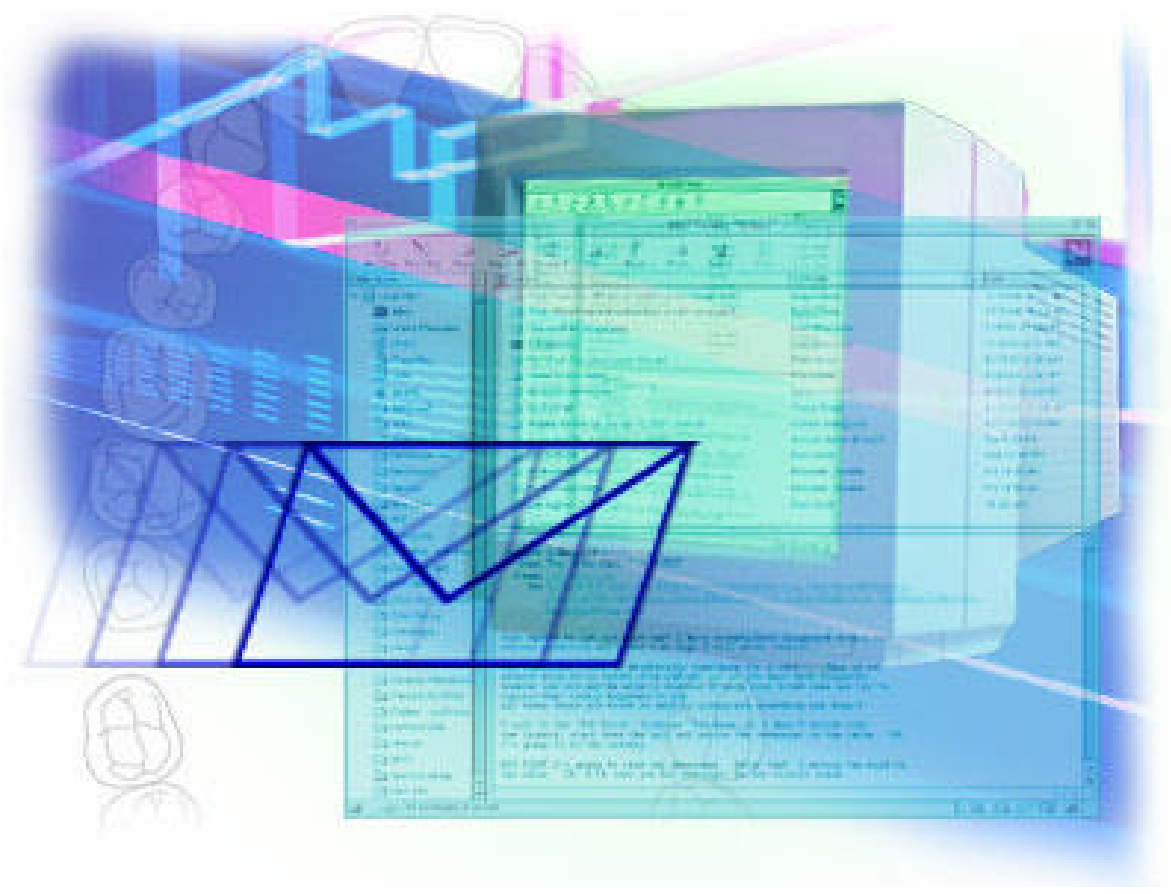

\section{Abstract}

E-mail is an increasingly common way to share information within business communities and the general population. This technology can significantly affect the process of and expectations for communications between the clinician and the patient. The unique characteristics and attributes of computer-based com munications can ultimately enhance outcomes for patient well-being if the clinician is informed, proactive, and avoids certain potential pitfalls related to the technology and its inclusion within the pattern of care.

In this article the author considers the impact of E-mail on personal and professional life and includes ideas the reader may find of value in managing and orchestrating this new dimension for communica tions.

Keywords: E-mail, "spamming," Electronic Communications Privacy Act, ECPA, HIPPA regulations 


\section{Introduction}

As the general public increasingly embraces the world of E-mail communication for both personal and professional conversations, E-mail is woven into the fabric of everyday life. For many, E-mail literally reaches across the globe to family, friends, and colleagues. Using the Internet, we connect to others, anywhere, anytime. The impact of E-mail on our lives can and should be incredibly productive and serves to enhance value in personal, professional, and business environments.

\section{The Burden and the Benefit}

As a communication tool, E-mail affords the user significant powers. Similar to the telephone it allows instantaneous delivery of information. However, unlike a telephone, it is never busy, does not require someone to answer, and allows access for minimal or often negligible cost. Email communication can also be managed asynchronously and responded to at the convenience of the recipient. Phone systems require voicemessaging services (often at increased cost) to provide similar functionality.

Although E-mail is transmitted in a similar manner to a telephone message, it can be better docu mented. Like postal mail it requires a unique address for both the recipient and the sender. Unlike postal mail, it is easily susceptible to inter ception or modification without the knowledge of the sender or the recipient.

E-mail can also be printed. This is an important convenience for practitioners using E-mail to manage patients and maintain a written patient treatment record. Because the functionality of Email depends on using the Internet, copies and records of the messages also reside on servers. Although the E-mail may be deleted from your computer, the electronic version will survive. Therefore, a user can neither deny sending or receiving a message. Because many copies of the message exist in many different environments as the message is transmitted over the Internet, E-mail also has the capacity to be shared (and viewed) beyond the immediate or designated recipient. Because E-mail is used to stay informed, stay in touch with others, and enhance the effectiveness and productivity of the work place, many view their initial introduction to Email as exhilarating and exciting. This excitement may be short-lived as overwhelming numbers of messages can, at times, arrive daily in the E-mail inbox. Often these messages are unsolicited and have little or no value. Worse yet, they all may require an immediate response. In this situation, dealing with E-mail is essentially like taking out the garbage or shoveling snow. It never ends and just keeps piling up until you do something about it.

Overwhelming, unpredictable, and unprecedented information overload is the true culprit. Modern society welcomes technology in an effort to increase efficiency and effectiveness. All too often, however, it finds itself drowning in a flood of useless knowledge. Information overload may come from commercial vendors using intelligence agents and data mining techniques in marketing to users and potential customers (e.g., Doubleclick.com). Innocent excess may even flow from the individual who makes it a mission to inform everyone about every detail of every aspect of every event with the simple stroke of the "Send" button.

\section{Broadcast Potential}

The broadcast potential of the E-mail message is enormous. Consider the case of Ms. Forrest's fifth grade class at Taylorsville Elementary School in North Carolina. Their curiosity initiated an experiment to see where in the world their E-mail would travel. The project "Geography Alive: Around the World with E-mail" started with the school's computer lab assistant sending copies of the E-mail to 32 people in her address book. A note asked these individuals to send the informa tion to everyone they knew online. Within an hour they heard from Texas, a couple of hours later Japan. In less than 30 days there were over ? million responses to the initial E-mail from all 50 states and over 70 foreign countries. ${ }^{1}$

The impact and potential power of geometric pro gression facilitated through technology is profoundly demonstrated in this example. There should be little doubt as to why marketers in the electronic world place such high value for elec tronic information networks. There is no other vehicle where marketers can generate such a vast connection with potential customers. Many states have instituted legislation that strictly regu lates the use of "spamming" or broadcasting unsolicited E-mail in order to protect the public from an onslaught of unwanted advertising. 
Rules of The Road

Solving time management needs for online resources on a personal level doesn't conclusive ly address the big-

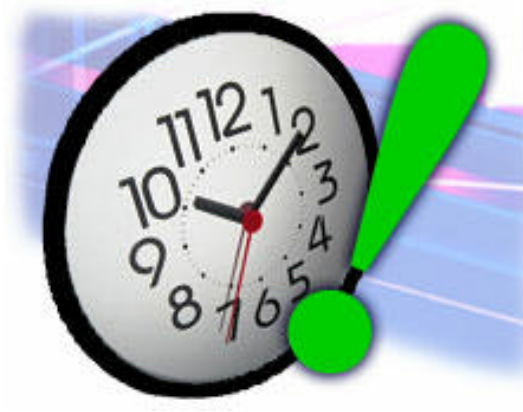
ger picture of functional (or dysfunctional) use of telecommunications. Effective utilization of E-mail for both business and personal functions extends beyond strategies that

relieve the congestion of the inbox. Because Email directly connects to the world at large, there are certain caveats and conventions that should be observed to better protect all E-mail corre spondents.

Effective use of rules of the road for E-mail requires using common sense and self-discipline. Charles Bermant, a writer for the Seattle Times, offers a thoughtful list of E-mail etiquette rules for online communication:

- Guard your E-mail address

- Get a Web based mailbox (e.g., hotmail)

-Don't share messages with shocking content

-Value your message (don't communicate excessively)

-Don't use the priority tag

-Don't reveal too much

-Respect the recipient

-Pay attention to (and effectively use) the "cc" and "re" lines

-Don't forward canned messages

-Say thank you

For a complete and unedited listing of E-mail Etiquette, access http://seattletimes.nwsource.com under "Personal Technology."2

\section{The Dreaded Virus}

Viruses are small computer programs that nefarious computer users send over networks with the intent of reaping havoc on users of E-mail. As a result, one should always take precautions when opening E-mail. It is advisable to exercise special caution before opening an attachment to an Email message from an unexpected, or an unknown source. Because virus attacks are increasingly frequent and sophisticated, it is not uncommon to inadvertently open an infected attachment, corrupting your computer and then unwittingly sharing the same fate with family, friends, and business contacts via subsequent Email communication spreading the virus. The more wide of E-mail becomes, the more potential exists for spreading computer viruses if caution is not exercised. Inexpensive anti-virus software programs are available (and recommended) to protect your computer against a virus attack and to repair files that may have been damaged by a virus (e.g., McAffee, Norton Antivirus). Some online E-mail sites (e.g., hotmail) scan all mes sages sent and received within their site with antivirus software and, therefore, protect their users' systems from importing "corrupt or tagged" data.

To learn more about the potential of virus/worm attacks to ruin computer systems, refer to McAfee, Symantec, and other virus bulletin boards (e.g., http://www.virusbtn.com/).

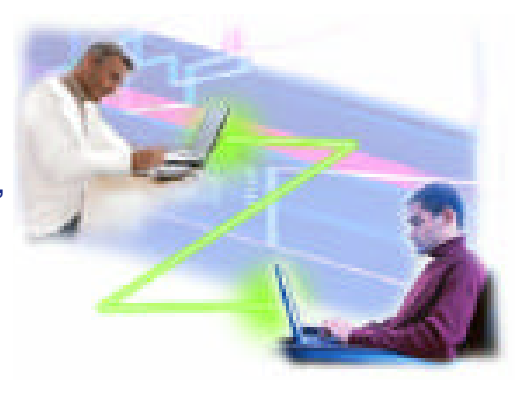

\section{E-mail Communication and Patient Care} While most clinicians still use postal mail and the telephone to manage both patient and inter-pro fessional communications in their dental practices, one to two percent of health practitioners have started to use E-mail for professional con nections with patients. ${ }^{3}$ More commonly, practitioners are starting to use E-mail to communicate with their colleagues. As the use of E-mail becomes more common in the office environ ment, practices should consider including the dis position of E-mail in the established and docu mented security policy of the office. 
Practitioners are also discovering the need to use the Internet to find and market to the "new con sumer" of the 21 st Century. ${ }^{4}$ Just as telephone came to the forefront as the convenience of the 20th Century, the landscape of technology utilization is migrating to new communications technolo gies for the 21st Century.

Clinicians may find complexity and frustration play a large part of their E-mail experience. Ironically, E-mail lurks as one of the most "time-devouring time-savers" of all time. It is so effective to "con nect," using E-mail has progressed to where it borders on counter-efficiency. E-mail takes on a life of its own and can overwhelm the activities and needs we once hoped it would sustain. The question is one of how to describe and implement solutions that tame the dragon.

\section{Change the Rules}

Effective measures can be taken to prevent the glut of E-mail information overload. Unfortunately the answer is not simplistic when taking strategic needs and responsibilities into account. This is especially true if professional interactions rely on electronic communication. Patients and physicians have certain rights and expectations when they communicate electronically. The practitioner who uses electronic communications should strictly adhere to privacy and confidentiality requirements as mandated by federal and state law and other regulations. Equal attention should be paid to exercising common courtesies and conventions traditionally used in conventional communications. For instance, the courtesy of a reply is a must. Cyberspace can be a wasteland of dead-ends and replies ignored because of missed and overlooked connections or information overload; so, the effective practitioner must engage effective protocols to effectively deal with such variables. Specific initiatives and strategies initiated in resolving these issues might include:

Divide and Conquer. Use folders in the E-mail software to divide content into more cognitively discernable and functional entities. Try establishing a dozen or so useful categories and set up folders for each of these groups. Move each meaningful message into an action-oriented group and expedite needed actions.

Don't Procrastinate. Deal with messages punctually. Sort the mail and answer what you can immediately. Keep the inbox small. Reduce the angst.
Use Routing Filters. Electronic filters can be used to automatically route specific types of Email messages to appropriate holding folders. For instance the subject line of incoming E-mail from patients can be set to read "patient E-mail." Software products such as Eudora or Outlook can file the message directly into a folder set up for that purpose. This can save time otherwise required to just sort messages based on the nature of message content.

The Delete Function. If the value of a message is not immediately clear, delete it. Revisit the delete folder later if it turns out to be important after all.

The Doctor is Out. Don't stay online continuously. Real E-mail junkies using an instant messaging service should give it up.

Remaining connected all the time generates more E-mail that others feel obliged to answer. This grows the quantity of unneeded E-mail. Save energy for the really important messages. Establish behavior (or a policy) that fits your needs and stick to it.

Sort the Wheat from the Chaff. Use the advan tages offered by E-mail programs. Sort and delete messages by the correspondent. Often times the information can be superfluous. Eliminate by reviewing the sequence of mes sages in context of the overall conversation. Flag the important messages that require more scruti ny during the process. Keep on track. Using the preview pane to instantly view content without actually opening the correspondence. This saves time, but beware, using the preview pane can make your system more susceptible to attack by an E-mail virus.

Multiple Addresses. Use different mailboxes based on "business." For instance, a personal Email address should be exclusive and different than the E-mail address for a practice. This also allows using a division of labor by assigning a staff member to review and sort E-mail corre spondence from patients, colleagues, and other business contacts. ${ }^{5}$ 
Add Functionality. Use intra office E-mail to augment your communications within the office. Some messaging systems (e.g., http://www.pinknotes.com) allow staff and practitioner to stay connected continuously throughout the day online. For those with integrated computer sys tems in the office this is a wonderfully progressive alternative to a light system or using "stickynotes."

\section{Special Healthcare Considerations}

These rules form a framework for online solutionoriented behavior. As a healthcare practitioner using E-mail for patient conversations, be informed and follow strict guidelines. Sharing personally identifiable healthcare information online with individuals or colleagues requires protecting the privacy of the individual's information. As discussed in the previous article of this series, ${ }^{6}$ federal legislative regulatory code addresses the privacy expectations of patients as they apply to the healthcare industry through the newly promulgated HIPAA regulations (Health Insurance Portability and Accountability Act of 1996). ${ }^{7}$

E-mail can be very useful to address patient com munication issues. Often, some characteristics inherent in the traditional office-based dental practice leave patients "wanting more."

Electronic contacts with practitioners can be less intimidating for some patients than in-person, face-to-face conversations. This technology certainly supports a "one-on-one" virtual opportunity to seek information and understand its implica tions for personal health outcomes.

Privacy is an issue in the day-to-day routines and protocols of healthcare. While increasing num bers of patients seek health information online, the practitioner must understand the implications for dental office practices and adapt protocols to address requirements and inform patients. The doctor-patient relationship implies certain expectations of privacy. New legislation (HIPAA) extends that responsibility to due diligence as information is shared outside of the doctor-patient relationship.

A cavalier attitude on the part of the clinician toward the use of E-mail for patient communica tion should be avoided. E-mail by definition is included as "all communication that is generated by or about the patient, including any photo graphs or imaging, is part of the medical record." ${ }^{8}$
As described by Attorney A. Spielberg writing in the Journal of the American Medical Association, "Medical E-mail represents a lasting record of healthcare provider judgment and patient response and the reporting of symptoms. Accordingly medical E-mail sent in a medical rela tionship, or generally in the course of treatment, should be drafted with care and composed as a formal written document on a professional letter head. Furthermore, as a precaution, the provider's preferred method of communication should be noted in the medical record, along with relevant telephone numbers and E-mail address es that the patient has specifically endorsed for use by healthcare practitioners for medical pur poses."

With the use of E-mail as a facilitator in health care practice, the patient needs to be informed about the level of privacy and confidentiality they might expect as their data is shared over vast institutional and private databases. Spielberg offers specific remedies in her paper for the American Medical Association. She says, "Medical E-mail should only be used after a patient has been informed of potential risks and benefits and signed a "E-mail consent" form. Despite arguments that a physician may infer consent if a patient initiates E-mail contact, physi cians should not assume that patients who use Email in other settings understand the implications of its use in the medical context." ${ }^{10}$

Additional concerns include the interception of Email containing medical data while transiting the Internet. Generally, the Electronic Communications Privacy Act (ECPA) of 1986 precludes the interception of data as it flows across a distributed network. However, because of the nature of the Internet and the server-router con figurations, static data is not protected by the ECPA. It is subject to archive and inspection by the transmitting service while it is housed or stored on their domain. ${ }^{11}$

In dentistry, these protections may seem excessive and overwhelming to implement. However, by taking the specific steps recommended by experts and required by law, the practitioner can illustrate concern for the patient and their right to a professional and confidential doctor-patient relationship. 


\section{Conclusion}

Using E-mail offers significant advantages in efficient communication. It can become burdensome if not managed properly. Special attention to legal considerations is required if E-mail is used by healthcare professionals to communicate with and about patients. By using common sense and forethought, E-mail can contribute productively to both professional and personal needs.

\section{References}

1. Schuessler H. Social Studies Class Finds How Far E-mail Travels, New York Times, Feb. 22, 2001.

2. Bermant C. http://seattletimes.nwsource.com/cgi-

bin/WebObjects/SeattleTimes.woa/wa/gotoArticle?zsection_id=268454832\&text_only=0\&slug=ptinbo18\&document_id=134268010, accessed 2.24.2001.

3. Ferguson T. Digital doctoring-opportunities and challenges in electronic patient-physician commu nication. JAMA. 1998 Oct 21;280(15):1361-2. No abstract available.

4. Borowitz SM, Wyatt JC. The origin, content, and workload of e-mail consultations. JAMA. 1998 Oct 21;280(15):1321-4.

5. Bermant C. Efficient Ways to Manage E-mail, Seattle Times, October 29,2000.

6. Day J., Privacy and Personal Health Data in Cyberspace, J Contemp Dent Pract, Winter, 2001, http://www.thejcdp.com, accessed 2.24.2001.

7. Health Insurance Portability and Accountability Act, Pub. L. 104-191, 42 USC 1320.

8. Spielberg A. Online without a Net, Physician-patient communication by electronic mail, American Journal of Law and Medicine, Summer-Fall 1999, p. 267.

9. Spielberg AR. On call and online: sociohistorical, legal, and ethical implications of e-mail for the patient-physician relationship. JAMA. 1998 Oct 21;280(15):1353-9.

10. See id.

11. Pub. L. No. 99-508, 100 Stat.1848 (1986).

\section{About the Author}

\section{James Day, DDS, MEd}

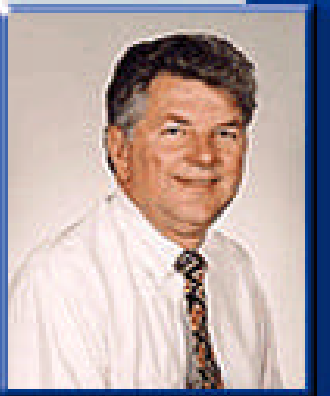

Dr. Day is a lecturer in the Division of Information Technology and Research in the Department of Oral Medicine at the University of Washington. He serves as the Vice-Chair of the American Dental Association ANSI Standards Committee for development of electronic technologies and Communication in dentistry and a member of the association's Task Force on Distance Learning. Correspondence related to this article and be sent to him at the following address:

Division of Information Technology and Research

Department of Oral Medicine

University of Washington School of Denstistry

\section{e-mail: jimyd@u.washington.edu}

